\title{
INEQUALITIES CONCERNING THE CHARACTERS OF A FINITE GROUP
}

\author{
K. L. FIELDS 1
}

ABSTRACT. Given a finite group we provide explicit bounds (in terms of the group order and numbers of conjugacy classes and involutions) for (a) the number of real valued characters of type $R$; (b) the sum of the degrees of the irreducible characters; (c) the sum of the entries of the character table; (d) the sums (b), (c) restricted to real valued characters. We al so provide a bound on the number of elements of order $2 n$ in terms of the number of elements of order $n$.

Let us first observe the following inequalities, which follow by application of the Cauchy-Schwarz inequality if one regards the summations on the left as inner products:

$$
\begin{gathered}
\sum_{x} \leq c^{1 / 2}\left(\sum n_{i}\right)^{1 / 2}, \\
\sum_{x \text { real }} a_{x} \leq k_{1}^{1 / 2}\left(\sum n_{i}\right)^{1 / 2}, \\
\left|\sum_{\chi}(a)\right| \leq c^{1 / 2}|C(a)|^{1 / 2}, \\
\left|\sum_{x \text { real }} \chi(a)\right| \leq k_{1}^{1 / 2}|C(a)|^{1 / 2}, \\
\sqrt{a} \leq k_{1}^{1 / 2}|C(a)|^{1 / 2} .
\end{gathered}
$$

Here $\sqrt{a}=\Sigma \epsilon(\chi) \chi(a)$ is the number of solutions in $G$ of $y^{2}=a ; \epsilon(\chi)=$ $0, \pm 1$, depending on whether the irreducible character $\chi$ is of type $\mathbf{C}, \mathbf{R}, \mathbf{H}$ (Frobenius-Schur [4]); $a_{\chi}$ is the sum of the elements in the $\chi$ th row of the character table; $|C(a)|=\Sigma a_{\chi} \chi(a)$ is the order of the centralizer of $a \in G[5]$; $c$ is the number of conjugacy classes; $k_{1}$ is the number of real conjugacy

Received by the editors February 8, 1974.

AMS (MOS) subject classifications (1970). Primary $20 \mathrm{C} 15$.

Key words and phrases. Characters of a finite group, positive semidefinite matrix.

1 We take this opportunity to thank the Department of Pure Mathematics and King's College, Cambridge for their generous hospitality during the author's tenure as a $\mathrm{Na}$ tional Science Foundation (NATO) Postdoctoral Fellow. 
classes; $n_{i}=\left|C\left(a_{i}\right)\right|$ where $a_{i}$ is an element of the $i$ th class $\left(a_{0}=e, n_{0}=\right.$ $g=|G|) ; m_{1}=\sqrt{e} ; l_{1}, l_{2}=k_{1}-l_{1}$ are the number of characters of type $\mathbf{R}, \mathbf{H}$ respectively; $d=\Sigma \chi(1) ; d_{1}=\Sigma_{\chi_{\text {real }}} \chi(1)$.

Observe that (4) and (5) both generalize a result of Brauer-Fowler, who prove a slightly stronger result when $a=e[2$, Theorem $2 \mathrm{~J}]$, although cf. Remark 1 below; and (1) and (2) frequently provide better upper bounds on the character sums than those of [3], [5].

All of these inequalities are contained in the following statement:

The real symmetric matrix

$$
M_{a}=\left[\begin{array}{ccccc}
\sum n_{i} & |C(a)| & \sum \epsilon(\chi) a_{\mathbf{x}} & \sum_{\mathbf{x} \text { real }} a_{\mathbf{x}} & \sum a_{\mathbf{x}} \\
|C(a)| & |C(a)| & \sqrt{a} & \sum_{\mathbf{x} \text { real }} \chi(a) & \sum \chi(a) \\
\sum \epsilon(\chi) a_{\mathbf{x}} & \sqrt{a} & k_{1} & l_{1}-l_{2} & l_{1}-l_{2} \\
\sum_{\mathbf{x} \text { real }} a_{\mathbf{x}} & \sum_{\mathbf{x} \text { real }} \chi(a) & l_{1}-l_{2} & k_{1} & k_{1} \\
\sum a_{\mathbf{x}} & \sum \chi(a) & l_{1}-l_{2} & k_{1} & c
\end{array}\right]
$$

is positive semidefinite.

For (1)-(5) merely state that certain of the $2 \times 2$ principal minors obtained by permuting corresponding columns and rows of $M_{a}$ are nonnegative (there are ten such minors altogether, but the other five lead to trivial or weaker inequalities than those above).

To see that $M_{a}$ is positive semidefinite, merely observe that $M_{a}=A_{a} A_{a}^{*}$ where

$$
A_{a}=\left[\begin{array}{ccc}
\cdots & a_{x} & \cdots \\
\cdots & \chi(a) & \cdots \\
\cdots & \epsilon(\chi) & \cdots \\
\cdots & \epsilon(\chi)^{2} & \ldots \\
\cdots & 1 & \ldots
\end{array}\right]
$$

is the $5 \times c$ matrix whose $\chi$ th column is as shown.

To obtain the inequalities which we desire, we permute corresponding rows and columns of $M_{a}$ and compute the $3 \times 3$ principal minors.

We should perhaps mention that we have here a veritable plethora of inequalities: first, for any $a \in G$ (and permutation of corresponding columns and rows) all of the principal minors of $M_{a}$ are nonnegative; we may also average the $M_{a}$ 's over subsets of $G$ to obtain positive semidefinite matrices; or we 
may average the principal minors of $M_{a}$ over subsets of $G$; or we may use Minkowski's result that the determinant function is concave on the set positive semidefinite matrices to obtain inequalities between the principal minors of the averaged $M_{a}$ 's and the average of the principal minors of $M_{a}$. We will content ourselves here, however, with five (of the ten) $3 \times 3$ principal minors of $M_{a}$ when $a=e$ :

$$
\begin{gathered}
c k_{1} g+2 d m_{1}\left(l_{1}-l_{2}\right)-m_{1}^{2} c-\left(l_{1}-l_{2}\right)^{2} g-d^{2} k_{1} \geq 0, \\
k_{1}^{2} g+2 d_{1} m_{1}\left(l_{1}-l_{2}\right)-m_{1}^{2} k_{1}-\left(l_{1}-l_{2}\right)^{2} g-d_{1}^{2} k_{1} \geq 0, \\
\left(\sum n_{i}\right) k_{1} g+2 m_{1} g \sum \epsilon(\chi) a_{\mathbf{X}}-m_{1}^{2} \sum n_{i}-k_{1} g^{2}-g\left(\sum \epsilon(\chi) a_{\mathbf{X}}\right)^{2} \geq 0, \\
\left(\sum n_{i}\right) k_{1} g+2 d_{1} g\left(\sum_{\mathbf{x} \text { real }} a_{\mathbf{x}}\right)-d_{1}^{2} \sum n_{i}-k_{1} g^{2}-g\left(\sum_{\mathbf{x} \text { real }} a_{\mathbf{x}}\right)^{2} \geq 0, \\
\left(\sum n_{i}\right) c g+2 d g\left(\sum a_{\mathbf{x}}\right)-d^{2} \sum n_{i}-c g^{2}-g\left(\sum a_{\mathbf{x}}\right)^{2} \geq 0 .
\end{gathered}
$$

From (6), (7) we obtain (after substituting $l_{2}=k_{1}-l_{1}$ )

Theorem 1.

(A)

$$
\begin{aligned}
\frac{k_{1}}{2}+\frac{m_{1} d_{1}}{2 g}- & \frac{1}{2 g} \sqrt{\left(g k_{1}-d_{1}^{2}\right)\left(g k_{1}-m_{1}^{2}\right)} \\
& \leq l_{1} \leq \frac{k_{1}}{2}+\frac{m_{1} d_{1}}{2 g}+\frac{1}{2 g} \sqrt{\left(g k_{1}-d_{1}^{2}\right)\left(g k_{1}-m_{1}^{2}\right)},
\end{aligned}
$$

$\left(A^{\prime}\right)$

$$
\frac{k_{1}}{2}+\frac{m_{1} d}{2 g}-\frac{1}{2 g} \sqrt{\left(g c-d^{2}\right)\left(g k_{1}-m_{1}^{2}\right)}
$$

$$
\leq l_{1} \leq \frac{k_{1}}{2}+\frac{m_{1} d}{2 g}+\frac{1}{2 g} \sqrt{\left(g c-d^{2}\right)\left(g k_{1}-m_{1}^{2}\right)},
$$

$$
d_{1} \leq m_{1} \frac{l_{1}-l_{2}}{k_{1}}+\sqrt{\left(g k_{1}-m_{1}^{2}\right)\left(k_{1}^{2}-\left(l_{1}-l_{2}\right)^{2}\right)}
$$

$$
d \leq m_{1} \frac{l_{1}-l_{2}}{k_{1}}+\sqrt{\left(g k_{1}-m_{1}^{2}\right)\left(c k_{1}-\left(l_{1}-l_{2}\right)^{2}\right)} .
$$

From (8), (9), (10), we obtain 
Theorem 2.

$$
\begin{aligned}
& \sum \epsilon(\chi) a_{\chi} \leq m_{1}+\left(\sum_{i \neq 0} n_{i}\right)^{1 / 2} \sqrt{k_{1}-m_{1}^{2} / g} \\
& \left|\sum_{\chi \text { real }} a_{\chi}-d_{1}\right| \leq\left(\sum_{i \neq 0} n_{i}\right)^{1 / 2} \sqrt{k_{1}-d_{1}^{2} / g}, \\
& \left|a_{x}-d\right| \leq\left(\sum_{i \neq 0} n_{i}\right)^{1 / 2} \sqrt{c-d^{2} / g} .
\end{aligned}
$$

We conclude with three remarks and an application:

1. Concerning $(\mathrm{A})$ and $\left(\mathrm{A}^{\prime}\right)$, one may estimate $d_{1}, d$ by $m_{1} \leq d_{1} \leq$ $k_{1}^{1 / 2} g^{1 / 2}, g^{1 / 2} \leq d \leq c^{1 / 2} g^{1 / 2}$ to obtain bounds on $l_{1}, l_{2}$ strictly in terms of $g, m_{1}$, $k_{1}, c$. In particular we have $m_{1}^{2} / g \leq l_{1} \leq k_{1}$ which sharpens Brauer-Fowler $[2$, Theorem $2 \mathrm{~J}]$ in another direction. We conjecture that $l_{1} \geq k_{1} / 2$; determining the exact relationship between $l_{1}, l_{2}$ and the internal structure $G$ is an old unsolved problem [1]. The upper bound in $(A)$ is attained whenever $k_{1}=$ $l_{1}$ (observe $m_{1}=d_{1}$ in this case); the upper bound in $\left(A^{\prime}\right)$ is attained whenever $c=k_{1}=l_{1}$. Note, however, that the upper bounds are also attained for the quaternion group of order 8 (where $l_{1}=k_{1}-1$ ).

2. Concerning (B) and (C), recall $m_{1} \leq d_{1} \leq d$ and, as in (A) and ( $\left.A^{\prime}\right)$, equality holds whenever $k_{1}=l_{1}$ (for (B)) or $c=k_{1}=l_{1}$ (for (C)). One may use the trivial estimate $\left|l_{1}-l_{2}\right| \leq k_{1}$ to obtain bounds strictly in terms of $g, m_{1}, k_{1}, c$.

3. Concerning (D), (E) and (F), recall that in [3] we proved that $\Sigma \epsilon(\chi) a_{\chi} \geq\left(m_{1}-1\right)+(c-r)+\left(k_{1}-k_{2}\right)$ so $\Sigma a_{\chi} \geq \Sigma \Sigma_{\text {real }} a_{\chi} \geq \Sigma \epsilon(\chi) a_{\chi} \geq m_{1}$. We conjecture that in fact $\Sigma a_{\chi} \geq d$ and $\Sigma_{\chi_{\text {real }}} a_{\chi} \geq d_{1}$. Observe that $\Sigma a_{\chi}-d$ is the sum of the elements of the character table outside of the first column; and $\Sigma_{\chi \text { real }} a_{\chi}-d_{1}$ is the sum of such elements in rows corresponding to real valued characters.

An application. Let us again consider the positive semidefinite matrix

$$
\left[\begin{array}{cc}
k_{1} & \sqrt{a} \\
\sqrt{a} & |C(a)|
\end{array}\right] .
$$

If we sum these matrices over the set of involutions of $G$ we obtain the positive semidefinite matrix

$$
\left[\begin{array}{cc}
m_{1} & m_{4} \\
m_{4} & g r
\end{array}\right]
$$


where $m$ denotes the number of involutions, $m_{4}$ the number of elements of order 4 , and $r$ the number of conjugacy classes of involutions. Hence $m_{4} \leq$ $\sqrt{g k_{1} m r}$. More generally,

Theorem 3. If $m_{n}$ denotes the number of elements of order $n$ and $r_{n}$ the number of conjugacy classes of elements of order $n$, then

$$
\begin{array}{ll}
m_{2 n} \leq \sqrt{g k_{1} m_{n} r_{n}} & \text { if } n \text { is even, } \\
m_{2 n} \leq \sqrt{g k_{1} m_{n}{ }^{r}}-m_{n} & \text { if } n \text { is odd. }
\end{array}
$$

When $n=1$ we have again the aforementioned result of Brauer-Fowler that $m \leq \sqrt{g k_{1}}-1$. Observe also that for the quaternion group of order 8 , $m_{4}=6$ and $g k_{1} m_{2} r_{2}=40$.

\section{BIBLIOGRAPHY}

1. R. Brauer, Representations of finite groups, Lectures on Modem Mathematics, vol. 1, Wiley, New York, 1963, pp. 133-175 (Problem 14). MR 31 \#2314.

2. R. Brauer and K. A. Fowler, On groups of even order, Ann. of Math. (2) 62 (1955), 565-583. MR 17, 580.

3. K. L. Fields, On the conjugating representation of a finite group, Proc. Amer. Math. Soc. 34 (1972), 35-37. MR 45 \#6941.

4. G. Frobenius and I. Schur, Über die Reellen Darstellungen der endlichen Gruppen, S.-B. Preuss. Akad. Wiss. 1906, 186-208.

5. L. Solomon, On the sum of the elements in the character table of a finite group, Proc. Amer. Math. Soc. 12 (1961), 962-963. MR 24 \#A2619.

DEPARTMENT OF MATHEMATICS, UNIVERSITY OF UTAH, SALT LAKE CITY, UTAH 84112 